\title{
Article \\ The Impact of COVID-19-Related Lockdown on Diet and Serum Markers in Healthy Adults
}

\author{
Nives Bogataj Jontez, Karin Novak (D), Saša Kenig (D), Ana Petelin, Zala Jenko Pražnikar and Nina Mohorko*D \\ Faculty of Health Sciences, University of Primorska, 6310 Izola, Slovenia; 97200376@student.upr.si (N.B.J.); \\ karin.novak@fvz.upr.si (K.N.); Sasa.Kenig@fvz.upr.si (S.K.); Ana.Petelin@fvz.upr.si (A.P.); \\ Zala.Praznikar@fvz.upr.si (Z.J.P.) \\ * Correspondence: nina.mohorko@fvz.upr.si; Tel.: +386-566-35801
}

Citation: Bogataj Jontez, N.; Novak, K.; Kenig, S.; Petelin, A.; Jenko Pražnikar, Z.; Mohorko, N. The Impact of COVID-19-Related Lockdown on Diet and Serum Markers in Healthy Adults. Nutrients 2021, 13, 1082. https://doi.org/ $10.3390 /$ nu13041082

Academic Editors: Carlo Agostoni and Gregorio Paolo Milani

Received: 4 March 2021

Accepted: 24 March 2021

Published: 26 March 2021

Publisher's Note: MDPI stays neutral with regard to jurisdictional claims in published maps and institutional affiliations.

Copyright: (C) 2021 by the authors. Licensee MDPI, Basel, Switzerland. This article is an open access article distributed under the terms and conditions of the Creative Commons Attribution (CC BY) license (https:/ / creativecommons.org/licenses/by/ $4.0 /)$.
Abstract: Due to limited data about the impact of lockdown on health status, the present study aimed to investigate the impact of COVID-19-related lockdown on changes in dietary habits, physical activity and serum markers in healthy adults. A total of 38 asymptomatic adults aged from 23 to 59 with a normal BMI $\left(22.5 \mathrm{~kg} / \mathrm{m}^{2}\right)$ participated in baseline and post-lockdown measurements that included dietary and physical activity assessment, anthropometric measurements and blood samples; and the lockdown survey which included dietary assessment and questionnaires about changes in lifestyle and physical activity. A decreased diet quality during lockdown was observed (Healthy Eating Index reduced from 64.59 to 61.08$)$, which returned to near baseline post-lockdown. Energy intake decreased during lockdown $(p=0.002)$ and returned to baseline post-lockdown. Despite lower physical activity levels during lockdown $(p=0.035)$, we observed no significant changes in body composition. However, we observed a significant increase in serum glucose $(p=0.005)$, total cholesterol $(p=0.003)$, and low-density lipoprotein (LDL) $(p=0.049)$ post-lockdown. Increase in serum glucose levels was pronounced in subjects with higher increase in energy intake $(p=0.039)$, increased omega- 6 fatty acids intake $(p=0.016)$, those who were exposed to several risky contacts ( $p=0.018$, compared to those with less risky contacts) and those who were not active in nature ( $p=0.008$, compared to those active in nature). Increased serum LDL was correlated to decreased monounsaturated fatty acids intake $(p=0.028)$. Within the limits of this preliminary report, changes in serum markers observed among healthy subjects point to a possible impact of COVID-19-related lockdown on adults' health to be confirmed in larger groups.

Keywords: lockdown; quarantine; nutrition; physical activity; serum biomarkers

\section{Introduction}

The ongoing coronavirus disease 2019 (COVID-19) outbreak has led to an unprecedented worldwide public health crisis. The COVID-19 outbreak was first reported in late December 2019 in Wuhan, Hubei Province, China. On 11 March 2020, the World Health Organization (WHO) declared the outbreak of COVID-19 to be a pandemic [1]. By the beginning of April 2020, half of the world population was in quarantine or lockdown that lasted until the end of April 2020 [2]. Isolation is different from lockdown as it is used only for those who are infected or sick [3]. The Slovenian government declared an epidemic status on 12 March 2020 [4] and on 16 March 2020 the gathering of people in public places was restricted and all unessential business was closed. The epidemic status ended on 15 May 2020 [5], but many restrictions remained. Lockdown or social distancing is an effective tool to prevent the spread of a new infectious disease [6], but affects lifestyle, including nutrition and physical activity. Evidence from recent studies suggests that pandemic-related coping strategies may have an adverse impact on health [7].

Behavioural changes occurred in a very short time. Restaurants, cafes, schools and kindergartens, gyms and recreational sport facilities were closed, and many people were 
working from home. Boredom, feelings of uncertainty, loss of freedom and separation from family and friends might have an impact on the psychological status of a person [3]. The increase in unstructured time, stress, and anxiety can further lead to overeating, sedentary behaviour and body mass gain [8]. Humans are sociable beings, so this period of being separated from family and friends caused a lot of people to eat more in quantity or frequency as a mechanism to cope with the growing fear and anxiety [9]. Fear of food shortage can lead to excessive food purchase and consumption [10] and in fact mass purchases of food took place in Slovenia, which was reported by the local media [11]. Mass purchases of food with a long shelf life, instead of fresh food, vegetables and fruit could lead to gaining body fat mass and a reduced intake of antioxidants. Additionally, lockdown restricted activities such as shopping, walking and playing outdoor games; sport facilities were also closed. As a result, many people avoided physical activity and lived a sedentary life. Insufficient physical activity leads to an increased risk of chronic noncommunicable diseases [3].

Consuming a well-balanced diet, focused on fruits and vegetables, wholegrains, plant and animal protein and unsaturated fatty acids leads to good health and normal immune function [9]. Higher fresh fruit and vegetables intake is linked to a higher intake of dietary fibre, vitamins, minerals and antioxidants. Adequate vitamin intake leads to a better immune defence and antioxidant intake improves low-grade inflammation [12] Diets rich in antioxidants (such as Mediterranean diet) are associated with a reduction in incidence and prevalence of chronic noncommunicable diseases such as cardiovascular disease, type 2 diabetes mellitus and cancer [10] which also seem to be a risk factor for the COVID-19 critical outcome. It is therefore crucial to maintain a good nutritional status and physical activity during periods with high infection risk.

Obesity, hypertension and insufficient physical activity, higher fasting serum glucose and higher total cholesterol levels have been identified by the WHO Global Health Observatory data as the most common and preventable risk factors for chronic noncommunicable diseases [13]. Increased waist circumference, increased serum triglyceride levels, reduced serum HDL levels, increased blood pressure and increased fasting serum glucose levels present the criteria for clinical diagnosis of metabolic syndrome [14] which is related to an increased risk for cardiovascular disease and type 2 diabetes mellitus.

To date, no studies have yet investigated the effects of lockdown on biochemical variables in healthy subjects. The aim of this study was to investigate changes in dietary habits, physical activity, and serum markers due to COVID-19 lockdown in Slovenia in a group of healthy lean subjects with interest in nutrition.

\section{Materials and Methods}

\subsection{Study Design}

The present study is a substudy of a larger study: The Link Between Diets and Health Indicators (DIETE) that started at the Faculty of Health Sciences, University of Primorska in Izola, Slovenia in December 2019 and was interrupted due to the COVID-19 pandemic. The study protocol was approved by the Slovenian National Medical Ethics Committee (No. 0120-557/2017/4) and was registered on ClinicalTrials.gov (Identifier: NCT04347213).

Baseline measurement (anthropometric measurements, blood sample, Food frequency questionnaire (FFQ), 3-day Food Diary, Lifestyle Questionnaire, International Physical Activity Questionnaire (IPAQ), Socio-Economic Questionnaire) took place in January, February and the beginning of March 2020 and was then interrupted due to the COVID-19 lockdown. After 4 weeks of lockdown, we invited all subjects with completed baseline measurement to participate in a survey on the effects of lockdown on their lifestyle (FFQ, IPAQ, Lifestyle and Socio-economic Changes Questionnaire). In the beginning of June 2020, they were invited to participate in a post-lockdown measurement (anthropometric measurements, blood sample, 3-day Food diary). All subjects were subjected to the same quarantine rules determined for the whole Slovene population that severely limited individual mobility, obliged everybody to work from home or refrain from work except from certain jobs, listed 
in the law as exceptions due to their importance for national security. Figure 1 shows the study design.

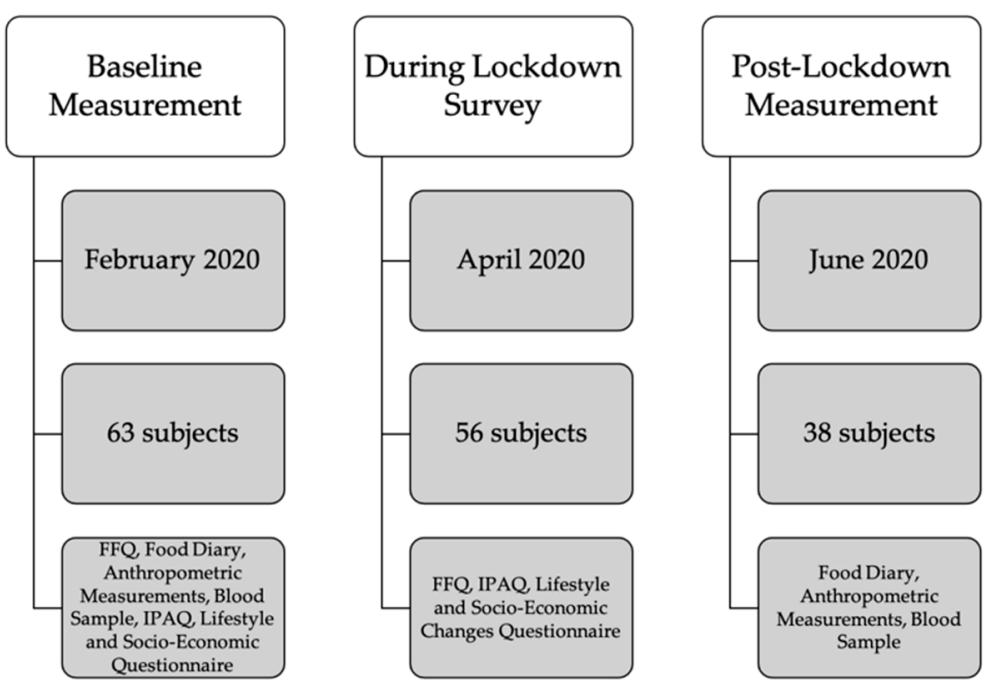

Figure 1. Study Design. FFQ—Food Frequency Questionnaire; IPAQ—International Physical Activity Questionnaire.

\subsection{Study Subjects}

Healthy lean volunteers with an interest in nutrition were recruited through a web survey posted on social media in groups dedicated to specific diet or healthy nutrition in December 2019. The inclusion criteria were body mass index (BMI) between $18.5 \mathrm{~kg} / \mathrm{m}^{2}$ and $30.0 \mathrm{~kg} / \mathrm{m}^{2}$, and age between 20 and 60 years. The exclusion criteria were any chronic disease, taking medications, being pregnant or lactating, a changed eating pattern 6 months preceding the inclusion in the study and a change in body mass (more than $3 \mathrm{~kg}$ ) 3 months preceding the inclusion in the study.

A total of 63 adults participated in the baseline measurement before the study was interrupted due to the pandemic. A total of 56 subjects agreed to take part in the lockdown survey. A total of 38 subjects agreed to participate in the post-lockdown measurements, 18 dropped out of the study, mainly for the fear of infection with SARS-CoV-2 while taking part in the measurements at our faculty.

\subsection{Dietary Assessment}

Subjects completed the validated FFQ for the Slovene population [15] and a questionnaire about the food-intake related habits at the baseline and four weeks into lockdown. The FFQ is a retrospective method for evaluating dietary intake in the last four weeks [15] and includes nine food groups: milk and dairy products, vegetables, fruit, starchy foods, legumes, meat and meat products, fat and fatty foods, sugar and beverages. It consists of eight frequency measures (never, once per month, 2 to 3 times per month, 1 to 2 times per week, 3 to 4 times per week, 5 to 6 times per week, 1 to 2 times per day, 3 or more times per day) and 3 portion sizes (small, medium, large). The subjects were provided with visualisation tools for more accurate portion assessment.

Subjects also completed a 3-day food diary at the baseline and post-lockdown. The subjects were instructed to record their food intake for three days in the same week (two weekdays and one weekend day). Where possible, subjects were asked to include food labels and recipes for mixed dishes. They were taught to weigh and record all food and beverages immediately before eating, and to weigh and describe any leftovers. They were also asked to report all food supplements taken that day.

Dietary data from the FFQ and food diary (energy, macronutrient, micronutrient intake and total- oxidative radical absorbance capacity (ORAC)) were analysed with the Open Platform for Clinical Nutrition (OPEN, http:/ / opkp.si/, accessed on 15 May 2020), 
a freely accessible online dietary assessment and planning tool that includes data from Slovenian nutrition tables [16], Souci Fachmann Kraut database [17] and United States National Nutrient Database for Standard Reference, available through the website http: / / www.ars.usda.gov/Services / docs.htm?docid=8964, accessed on 15 May 2020.

Diet quality was evaluated by calculating the Healthy Eating Index 2015 (HEI) [18] according to the developer's protocol [19]. The HEI includes 13 categories: total fruits, whole fruits, total vegetables, greens and beans, whole grains, dairy, total protein foods, seafood and plant protein foods, fatty acids ratio (polyunsaturated and monounsaturated fatty acids/saturated fatty acids), refined grains, sodium, added sugars and saturated fats, all estimated per energy intake unit $(1000 \mathrm{kcal})$. The first nine categories are scored positively and the last four categories are scored negatively from zero to five or to ten points proportionally to the interval between standards, as specified by Center for Nutrition Policy and Promotion [19]. The HEI (0-100) is the sum of points from all categories with higher score representing a higher diet quality.

The HEI was calculated from food diary data on baseline and post-lockdown and from FFQ during lockdown. To evaluate whether the HEI calculated from food diary and FFQ could be compared, HEI validation was performed using FFQ and food diary data at baseline from 17 subjects. Normal data distribution was determined with the Shapiro-Wilk test. Pearson correlation was used to establish the correlation between both methods $(\mathrm{r}=0.674, p=0.003)$. Student's T-test did not show any statistically significant differences between the two methods $(p=0.914)$, which implies both methods are equally reliable for HEI evaluation.

\subsection{Lifestyle and Socio-Economic Questionnaire}

At baseline, subjects were asked to report their education, marital status, work, socioeconomic status, living arrangement, and smoking and alcohol consumption habits. As part of the lockdown survey, they were inquired about the changes in previously reported data during lockdown, and about the changes in working conditions and workload. To assess the impact of the pandemic on their quality of life, they were asked about the frequency of following the news, whether they perceived changes in lifestyle and quality of life during lockdown, how frequently they visited the grocery store and about the changes of outdoor activities. We also inquired whether they respected social distancing and hygiene measures adopted by the government and whether they contracted COVID-19.

\subsection{Physical Activity Questionnaire}

The IPAQ [20] was used to calculate the physical activity-induced energy expenditure. The IPAQ includes questions about work-related physical activity, activity due to transport and physical activity during free time. Each question consists of the duration and intensity of the activity. Data from the duration and intensity were used to calculate daily physical induced energy expenditure in the metabolic equivalent of the task (MET).

\subsection{Anthropometric Measurements}

Anthropometric measurements were performed at baseline and post-lockdown following an overnight fast (at least $12 \mathrm{~h}$ ) in standardized conditions with light clothing and without shoes by the same examiner. Body mass, body fat percentage, fat mass, lean mass, muscle mass, visceral index, total body water, extracellular water, intracellular water and phase angle were measured using a bioelectric impedance analyser Tanita BC 418MA (Tanita Corporation, Arlington Heights, IL, USA).

\subsection{Serum Markers}

Venous blood samples were collected in $5 \mathrm{~mL}$ serum vacuum blood collection tubes at baseline and post-lockdown following an overnight fast. Samples were set to clot at room temperature for 30-60 min in line with Standard operating procedures for serum and plasma collection [21] and then centrifuged at $2000 \mathrm{rpm}$ for $10 \mathrm{~min}$ on room temperature. 
Serum was immediately separated, frozen and stored at $-80{ }^{\circ} \mathrm{C}$ until subsequent analysis. Serum glucose, triglycerides, total cholesterol, low-density lipoprotein (LDL), high-density lipoprotein (HDL), iron, aspartate transaminase (AST), bilirubin and C-reactive protein (CRP) levels were determined with a Cobas c111 analyser (Roche, Basel, Switzerland) using the specific Cobas c111 reagent for each parameter (Roche). Total cholesterol/HDL ratio was calculated for each individual.

\subsection{Statistical Analysis}

Statistical analysis was performed using IBM SPSS Statistics 26.0 (IBM, Armonk, NY, USA). Means and standard deviations were calculated. The normality of data distribution was evaluated using the Shapiro-Wilk test. Student's paired samples t-test or one-way ANOVA were used to compare the effect of lockdown between two or more normally distributed variables, respectively, while two or more non-normally distributed variables were compared with Wilcoxon's signed-rank test or Kruskall-Wallis' test, respectively. Repeated measures ANOVA was used to investigate changes in diet quality from baseline through lockdown to post-lockdown for normally distributed data, for non-normally distributed data Friedman's test was used. Changes of post-lockdown data from baseline $(\Delta)$ for nutritional intake and serum markers were calculated by subtracting baseline values from post-lockdown values. Pearson's Correlation was used to investigate associations between $\Delta$ nutritional intake and $\Delta$ serum markers for normally distributed data, for non-normally distributed data Spearman's Correlation was used. $p$-values $<0.05$ were considered statistically significant.

Post hoc, statistical power of the study was calculated based on primary outcome, $\Delta$ serum glucose, using G*Power 3.1.9.7 (Heinrich-Heine-Universität Düsseldorf, Germany), assuming an $\alpha$ level of $5 \%$ and $\beta$ level of $20 \%$. Effect size for $\mathrm{N}=38$ was calculated.

\section{Results}

\subsection{Subjects' Baseline Characteristics}

Subjects' baseline characteristics are reported in Table 1. The final sample included 14 male and 24 female Caucasian subjects, aged from 23 to 59 years with a normal BMI. The subjects were healthy, asymptomatic adults with stable body mass and stable eating pattern in the last 6 months. A total of $83.8 \%$ of the subjects had at least a bachelor's degree. The majority $(81.1 \%)$ of the subjects were married or in a non-marital partnership and only $10.8 \%$ of the subjects were living alone. A total of $67.6 \%$ of the subjects never smoked, while only $16.2 \%$ of them never drank alcohol. A total of $55.3 \%$ of the subjects consumed meat, while $44.7 \%$ of them consumed a plant-based diet. The analysis did not show any statistically significant differences in diet quality between meat-eaters and nonmeat eaters.

\subsection{Lifestyle and Socio-Economic Changes during Lockdown}

Table 2 reports the lifestyle changes during lockdown. Almost all subjects (94.6\%) stated that their lifestyle changed during lockdown and more than half $(59.5 \%)$ of the subjects stated they were following more news than usual. None of the subjects had contracted COVID-19. Only a third of the subjects (33.3\%) experienced no changes in working conditions during lockdown, while others were not working or working (at least partially) from home. Almost three quarters (73.0\%) of the subjects were active in nature and woods, while others were active only in close proximity to home during lockdown. Of the subjects who were working during lockdown, around half (48.1\%) were physically active the same as before during their work time, while others were less active or could not specify an answer. 
Table 1. Study Subjects' Baseline Characteristics.

\begin{tabular}{|c|c|}
\hline Characteristics & Mean \pm SD \\
\hline $\mathrm{n}$ & 38 \\
\hline Age (years) & $36.3 \pm 10.1$ \\
\hline Height $(\mathrm{cm})$ & $170.8 \pm 8.3$ \\
\hline Body mass (kg) & $65.8 \pm 10.2$ \\
\hline $\operatorname{BMI}\left(\mathrm{kg} / \mathrm{m}^{2}\right)$ & $22.5 \pm 2.7$ \\
\hline Gender & $\%$ \\
\hline Female & 63.2 \\
\hline Male & 36.8 \\
\hline Education & $\%$ \\
\hline High school diploma & 16.2 \\
\hline Bachelor's degree & 70.3 \\
\hline Master's degree or PhD & 13.5 \\
\hline Marital Status & $\%$ \\
\hline Single & 18.9 \\
\hline In a relationship or married & 81.1 \\
\hline Living Arrangement & $\%$ \\
\hline Alone & 10.8 \\
\hline With partner & 35.1 \\
\hline With partner and children & 40.5 \\
\hline With parents & 13.5 \\
\hline Smoking & $\%$ \\
\hline Never & 67.6 \\
\hline Occasionally & 24.3 \\
\hline Regularly & 8.1 \\
\hline Alcohol Consumption & $\%$ \\
\hline Never & 16.2 \\
\hline Occasionally & 75.7 \\
\hline Regularly & 5.4 \\
\hline Meat Consumption & $\%$ \\
\hline Regular meat consumption & 55.3 \\
\hline No meat consumption & 44.7 \\
\hline
\end{tabular}

Table 2. Lifestyle Changes During Lockdown.

\begin{tabular}{cc}
\hline General Lifestyle Changes during Lockdown & $\%$ \\
\hline Lifestyle did not change at all & 5.4 \\
Lifestyle changed a little & 56.8 \\
Lifestyle changed a lot & 32.4 \\
Lifestyle changed completely & 5.4 \\
No changes & $\%$ \\
Changes in working conditions during lockdown & 33.3 \\
Work from home, previously partially worked from home & 11.1 \\
Work from home, previously never worked from home & 13.9 \\
Partially work from home and partially at the workplace & 11.1 \\
Not working, receiving compensation & 22.2 \\
Not working, not receiving compensation & 8.3 \\
\hline Changes in socio-economic status during lockdown & $\%$ \\
Received 0-50\% of baseline monthly income & 16.2 \\
Received 80\% of baseline monthly income & 16.2 \\
Received 90-100\% of baseline monthly income & 67.6 \\
\hline Visiting the grocery store during lockdown & $\%$ \\
Less than once per week & 35.1 \\
Once per week & 48.6 \\
Twice per week & 13.5 \\
More than twice per week & 2.7 \\
\hline Activities outdoors during lockdown & $\%$ \\
Activities in close proximity to home & $27.0 \%$ \\
Activities in the nature and woods & $73.0 \%$ \\
\hline
\end{tabular}




\subsection{Changes in Nutrition and Physical Activity during and Post-Lockdown}

Subjects consumed on average $3.53 \pm 1.03$ meals per day at baseline and $3.33 \pm 0.96$ meals per day during lockdown. The change was not significant $(p=0.127)$.

The HEI dropped significantly during lockdown and improved post-lockdown but did not reach the baseline value (Table 3). Vegetable, greens and beans, protein and seafood and plant protein intake per energy unit and fatty acids intake ratio (polyunsaturated fatty acids [PUFA] + monounsaturated fatty acids [MUFA])/saturated fatty acids [SFA]) significantly decreased throughout the study. In contrast, dairy and refined grains intake per energy unit significantly increased throughout the study. Added sugar intake per energy unit increased during lockdown but decreased post-lockdown. Sodium intake per energy unit was significantly higher post-lockdown. Physical activity induced energy expenditure significantly dropped during lockdown from $13.86 \pm 22.48$ MET at baseline to $9.89 \pm 13.62$ MET during lockdown $(p=0.035)$.

Table 3. Energy Intake and Healthy Eating Index and its Categories at Baseline, During Lockdown and Post-Lockdown.

\begin{tabular}{|c|c|c|c|c|}
\hline & Baseline & Lockdown & Post-Lockdown & $p$ Value * \\
\hline Energy intake (kcal) & $2296.78 \pm 873.26$ & $1891.03 \pm 678.13^{\mathrm{a}}$ & $2248.17 \pm 576.76^{c}$ & 0.002 \\
\hline Total fruits (g/1000 kcal) & $109.79 \pm 102.00$ & $113.57 \pm 101.45$ & $108.75 \pm 67.38$ & 0.916 \\
\hline Whole fruits (g/1000 kcal) & $85.77 \pm 78.25$ & $90.51 \pm 78.49$ & $83.13 \pm 62.66$ & 0.829 \\
\hline Total vegetables (g/1000 kcal) & $507.16 \pm 377.07$ & $581.21 \pm 411.26$ & $134.05 \pm 92.13^{b, c}$ & $<0.001$ \\
\hline Greens and beans (g/1000 kcal) & $76.57 \pm 113.65$ & $54.04 \pm 57.01$ & $22.39 \pm 23.31^{b, c}$ & 0.002 \\
\hline Whole grains (g/1000 kcal) & $23.14 \pm 24.75$ & $21.11 \pm 24.41$ & $28.85 \pm 29.30$ & 0.319 \\
\hline Dairy (g/1000 kcal) & $60.70 \pm 69.39$ & $64.65 \pm 67.59$ & $172.98 \pm 143.27^{b, c}$ & $<0.001$ \\
\hline Total protein food (g/1000 kcal) & $88.95 \pm 66.92$ & $69.31 \pm 46.38^{a}$ & $54.55 \pm 33.47^{\mathrm{b}}$ & 0.002 \\
\hline Seafood and plant proteins $(\mathrm{g} / 1000 \mathrm{kcal})$ & $49.27 \pm 39.97$ & $35.37 \pm 28.92^{\mathrm{a}}$ & $29.91 \pm 22.52^{b}$ & 0.002 \\
\hline Fatty acids (PUFA+MUFA)/SFA) & $1.98 \pm 1.34$ & $1.77 \pm 1.20^{\mathrm{a}}$ & $1.54 \pm 0.78^{b}$ & 0.026 \\
\hline Refined grains (g/1000 kcal) & $29.67 \pm 28.17$ & $39.81 \pm 37.58^{\mathrm{a}}$ & $52.72 \pm 48.19^{b}$ & 0.006 \\
\hline Sodium (g/1000 kcal) & $0.95 \pm 0.44$ & $1.12 \pm 0.73$ & $1.19 \pm 0.54^{b}$ & 0.071 \\
\hline Added sugar (\%EI) & $10.93 \pm 6.08$ & $12.39 \pm 6.67$ & $4.84 \pm 3.93^{b, c}$ & $<0.001$ \\
\hline Saturated fats (\%EI) & $10.98 \pm 7.46$ & $11.82 \pm 8.47$ & $13.23 \pm 8.79^{b, c}$ & 0.001 \\
\hline HEI & $64.59 \pm 15.76$ & $61.08 \pm 13.42^{\mathrm{a}}$ & $63.26 \pm 15.22$ & 0.203 \\
\hline
\end{tabular}

HEI-Healthy Eating Index. * - repeated measures ANOVA or Friedman's test. a $-p<0.05$, Student's or Wilcoxon's signed-rank test between baseline and lockdown; ${ }^{\mathrm{b}}-p<0.05$, Student's or Wilcoxon's signed-rank test between baseline and post-lockdown; ${ }^{\mathrm{c}}-p<0.05$, Student's or Wilcoxon's signed-rank test between lockdown and post-lockdown measurement.

Although there were no statistically significant differences between baseline and post-lockdown HEI and energy intake, some significant differences in dietary intake were observed (Table 4). Energy density and omega-6 fatty acids intake significantly decreased, whereas the intake of fibre, saturated fatty acids and starch significantly increased. The intake of some micronutrients, such as riboflavin, niacin, folate, magnesium and copper also increased and total-ORAC also increased post-lockdown.

\subsection{Anthropometric Characteristics}

Subjects reported no changes in body mass during lockdown, which was confirmed in the post-lockdown measurement. There were no statistically significant changes in anthropometric variables post-lockdown (Table 5).

\subsection{Significant Changes in Serum Markers Post-Lockdown}

We observed a statistically significant increase in serum glucose $(p=0.005)$, total cholesterol $(p=0.003)$ and LDL $(p=0.049)$ levels, and a statistically significant decrease in serum CRP levels $(p=0.008)$ post-lockdown (Table 6). Total cholesterol/HDL ratio significantly increased from $2.760 \pm 1.171$ at baseline to $3.871 \pm 3.427$ post lockdown $(\mathrm{Z}=4.358, p<0.001)$. Post hoc calculated effect size for $\mathrm{N}=38$ for $\Delta$ serum glucose $(0.361 \pm 0.746)$ was 0.483 and the power of two tailed Student's test was 0.826. 
Table 4. Energy, Macronutrient and Micronutrient Intake from Diet at Baseline and Post-Lockdown.

\begin{tabular}{|c|c|c|c|}
\hline Variable (Unit) & Baseline & Post-Lockdown & $p$ Value * \\
\hline Energy density $(\mathrm{kcal} / \mathrm{g}) *$ & $1.15 \pm 0.32$ & $0.81 \pm 0.27$ & 0.000 \\
\hline Protein $(\mathrm{g})$ & $86.47 \pm 36.45$ & $87.25 \pm 36.45$ & 0.567 \\
\hline Carbohydrate (g) & $241.18 \pm 151.18$ & $249.45 \pm 131.06$ & 0.456 \\
\hline Fat $(\mathrm{g})$ & $89.29 \pm 35.63$ & $93.01 \pm 41.82$ & 0.345 \\
\hline Dietary fibre $(\mathrm{g})$ * & $28.35 \pm 17.30$ & $31.49 \pm 17.95$ & 0.046 \\
\hline Dietary fibre $(\mathrm{g} / 1000 \mathrm{kcal})$ * & $12.81 \pm 6.45$ & $14.15 \pm 7.82$ & 0.031 \\
\hline Soluble fibre $(g)$ & $5.54 \pm 4.49$ & $6.02 \pm 3.84$ & 0.272 \\
\hline Unsoluble fibre (g) & $10.93 \pm 8.70$ & $12.23 \pm 8.18$ & 0.197 \\
\hline Sugar $(g)$ & $80.62 \pm 55.72$ & $76.70 \pm 48.44$ & 0.690 \\
\hline Free sugar $(\mathrm{g})$ & $33.53 \pm 29.16$ & $29.70 \pm 27.88$ & 0.464 \\
\hline $\operatorname{Starch}(g) *$ & $68.13 \pm 62.95$ & $84.17 \pm 58.09$ & 0.048 \\
\hline Plant protein $(\mathrm{g})$ & $37.26 \pm 24.89$ & $40.82 \pm 24.46$ & 0.065 \\
\hline Saturated fatty acids $(\mathrm{g})^{*}$ & $26.54 \pm 18.82$ & $31.76 \pm 20.68$ & 0.003 \\
\hline Monounsaturated fatty acids (g) & $28.55 \pm 14.23$ & $27.07 \pm 12.73$ & 0.378 \\
\hline Polyunsaturated fatty acids (g) & $13.25 \pm 7.84$ & $12.43 \pm 6.10$ & 0.421 \\
\hline Omega-6 fatty acids $(\mathrm{g}) *$ & $7.29 \pm 5.95$ & $6.05 \pm 4.26$ & 0.042 \\
\hline Omega-3 fatty acids (g) & $1.45 \pm 1.59$ & $1.50 \pm 1.26$ & 0.357 \\
\hline Omega-3/omega-6 ratio * & $0.27 \pm 0.39$ & $0.29 \pm 0.18$ & 0.023 \\
\hline Cholesterol (mg) & $323.35 \pm 376.50$ & $316.96 \pm 356.47$ & 0.328 \\
\hline Vitamin C (mg) & $135.40 \pm 99.23$ & $110.94 \pm 70.30$ & 0.232 \\
\hline Vitamin D $(\mu g)$ & $4.08 \pm 3.45$ & $4.61 \pm 5.08$ & 0.637 \\
\hline Vitamin E (mg) & $12.51 \pm 7.72$ & $10.83 \pm 4.78$ & 0.255 \\
\hline Riboflavin-vitamin $\mathrm{B}_{2}(\mathrm{mg})$ * & $1.65 \pm 0.62$ & $2.00 \pm 0.76$ & 0.003 \\
\hline Niacin-vitamin $\mathrm{B}_{3}(\mathrm{mg})$ * & $20.10 \pm 10.99$ & $29.56 \pm 12.26$ & 0.000 \\
\hline Biotin-vitamin $B_{7}(\mu \mathrm{g})$ & $35.78 \pm 20.41$ & $38.97 \pm 19.10$ & 0.076 \\
\hline Folate $(\mu g) *$ & $360.91 \pm 175.88$ & $442.64 \pm 225.83$ & 0.001 \\
\hline Vitamin $B_{12}(\mu \mathrm{g})$ & $5.02 \pm 6.62$ & $5.70 \pm 13.54$ & 0.983 \\
\hline Calcium (mg) & $855.04 \pm 373.67$ & $938.57 \pm 468.35$ & 0.421 \\
\hline Iron (mg) & $17.23 \pm 7.38$ & $18.24 \pm 6.55$ & 0.210 \\
\hline Magnesium (mg) * & $407.79 \pm 224.65$ & $560.13 \pm 224.44$ & 0.000 \\
\hline Zinc (mg) & $10.56 \pm 5.35$ & $11.54 \pm 4.46$ & 0.062 \\
\hline Copper $(\mu g) *$ & $1865.12 \pm 923.61$ & $2429.81 \pm 1144.20$ & 0.001 \\
\hline Alcohol (g) & $6.41 \pm 11.96$ & $5.88 \pm 10.02$ & 0.387 \\
\hline Total-ORAC $(\mu \mathrm{mol} / \mathrm{TE}) *$ & $8346.48 \pm 6271.20$ & $10874.57 \pm 6949.49$ & 0.005 \\
\hline
\end{tabular}

* $p<0.05$; Total-ORAC — total oxidative radical absorbance capacity of daily food intake.

Table 5. Anthropometric Variables of Asymptomatic Subjects at the Baseline and Post-Lockdown.

\begin{tabular}{cccc}
\hline & Baseline & Post-Lockdown & $p$ Value \\
\hline Body mass (kg) & $65.8 \pm 10.2$ & $66.3 \pm 10.5$ & 0.342 \\
Body fat percentage (\%) & $22.2 \pm 7.6$ & $22.4 \pm 7.2$ & 0.639 \\
Fat mass (kg) & $14.7 \pm 5.5$ & $14.8 \pm 5.2$ & 0.711 \\
Fat free mass (kg) & $51.1 \pm 8.9$ & $51.4 \pm 9.6$ & 0.400 \\
Muscle mass (kg) & $48.5 \pm 8.5$ & $48.8 \pm 9.1$ & 0.442 \\
BMI (kg/m $\left.{ }^{2}\right)$ & $22.5 \pm 2.7$ & $22.7 \pm 2.6$ & 0.413 \\
Visceral index & $3.8 \pm 2.3$ & $3.7 \pm 2.3$ & 0.059 \\
Total body water (kg) & $36.6 \pm 6.3$ & $56.8 \pm 6.8$ & 0.695 \\
Total body water (\%) & $55.8 \pm 6.2$ & $6.00 \pm 0.6$ & 0.987 \\
Phase angle & $6.00 \pm 0.7$ & 0.883 \\
\hline
\end{tabular}

BMI—body mass index.

To assess whether the changes in serum markers and dietary intake per energy unit at post-lockdown from baseline differed among different lifestyle circumstances during lockdown, we divided the participants into groups based on reported lifestyle factors during lockdown (Figure 2). Subjects that were active only in close proximity to home had a significantly higher increase in serum glucose levels than subjects that were active in nature or woods $(Z=2.646, p=0.008)$. A trend of increased serum total cholesterol and LDL levels is also visible, but the changes were not significant (Figure 2a). Subjects that frequently or regularly consumed alcohol during lockdown had a significantly higher increase in serum triglyceride levels than subjects that never or rarely consumed alcohol $(Z=-2.417$, $p=0.016)$. A trend of increased serum total cholesterol and LDL levels is also visible, but 
the changes were not significant (Figure $2 b$ ). Subjects that were interacting socially with work colleagues or family and friends outside their household had a significantly higher increase in serum glucose levels than subjects who were interacting socially only with family in the same household $\left(\chi^{2}=8.008, p=0.018\right)$. A trend of increased serum total cholesterol levels is also visible, but the changes were not significant (Figure 2c). Subjects who were worried or very worried about infection with SARS-CoV-2 had a significantly higher increase in serum AST levels $(Z=-2.501, p=0.012)$ than subjects who were mostly not worried about infection. A trend of increased serum glucose, total cholesterol and LDL levels is also visible, but the changes were not significant (Figure 2d).

Table 6. Serum Markers in Asymptomatic Subjects at the Baseline and Post-Lockdown.

\begin{tabular}{|c|c|c|c|c|c|}
\hline & Baseline & Post-Lockdown & $p$ Value * & Slovenian Reference Values & $\Delta$ \\
\hline Glucose $(\mathrm{mmol} / \mathrm{L})$ * & $4.850 \pm 0.425$ & $5.210 \pm 0.698$ & 0.005 & $3.6-6.1$ & $0.361 \pm 0.746$ \\
\hline Total cholesterol $(\mathrm{mmol} / \mathrm{L})$ * & $5.447 \pm 3.523$ & $5.947 \pm 3.445$ & 0.003 & $4.0-5.2$ & $0.500 \pm 0.985$ \\
\hline $\operatorname{HDL}(\mathrm{mmol} / \mathrm{L})$ & $2.008 \pm 0.402$ & $2.075 \pm 0.592$ & 0.375 & $>1.4$ & $0.067 \pm 0.459$ \\
\hline $\operatorname{LDL}(\mathrm{mmol} / \mathrm{L}) *$ & $3.852 \pm 3.614$ & $4.102 \pm 3.560$ & 0.049 & $2.0-3.3$ & $0.250 \pm 0.815$ \\
\hline Triglycerides (mmol/L) & $0.953 \pm 0.574$ & $1.138 \pm 0.640$ & 0.054 & $0.6-1.7$ & $0.186 \pm 0.679$ \\
\hline $\mathrm{CRP}(\mathrm{mg} / \mathrm{L})$ * & $0.941 \pm 1.315$ & $0.635 \pm 1.108$ & 0.008 & $0.0-8.0$ & $-0.306 \pm 1.591$ \\
\hline Iron $(\mu \mathrm{mol} / \mathrm{L})$ & $23.263 \pm 10.663$ & $25.905 \pm 9.850$ & 0.283 & M 10.6-28.3, W 6.6-26.0 & $2.642 \pm 11.887$ \\
\hline AST (U/L) & $22.150 \pm 6.606$ & $23.613 \pm 10.701$ & 0.658 & $\begin{array}{l}\mathrm{M}<34.8 \\
\mathrm{~W}<31.2\end{array}$ & $1.463 \pm 10.270$ \\
\hline Total bilirubin $(\mu \mathrm{mol} / \mathrm{L})$ & $8.405 \pm 5.369$ & $9.768 \pm 5.422$ & 0.066 & $0.0-17.0$ & $1.363 \pm 4.330$ \\
\hline
\end{tabular}

${ }^{*} p<0.05$ is considered statistically significant; HDL-High-density lipoprotein; LDL-Low-density lipoprotein; CRP-C-reactive protein, AST-Aspartate transaminase; $\mathrm{M}-$ men, $\mathrm{W}-$ women, $\Delta-$ changes of post-lockdown data from baseline.

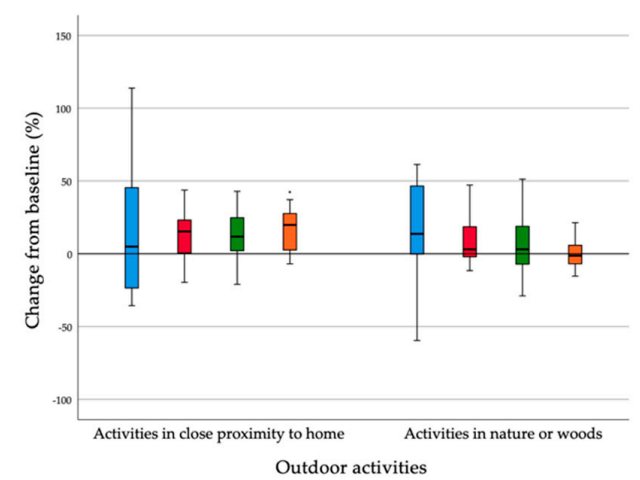

(a)

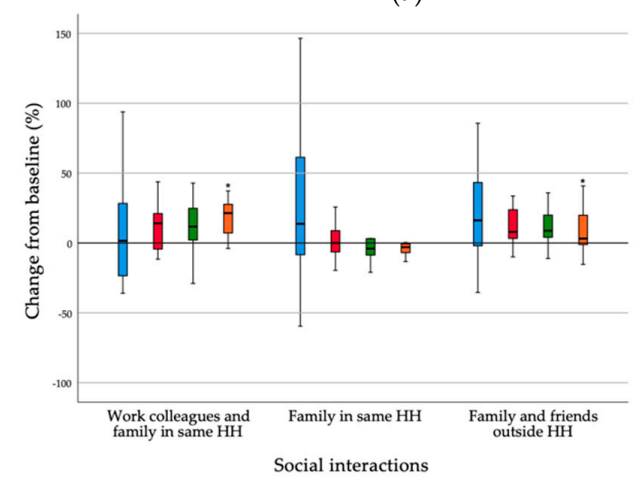

(c)
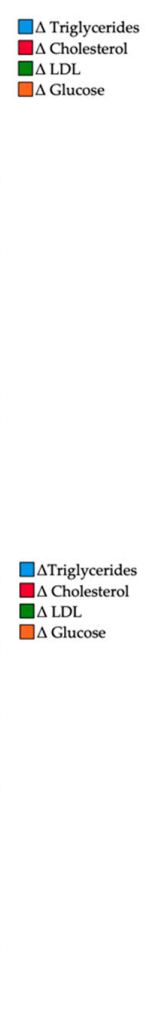

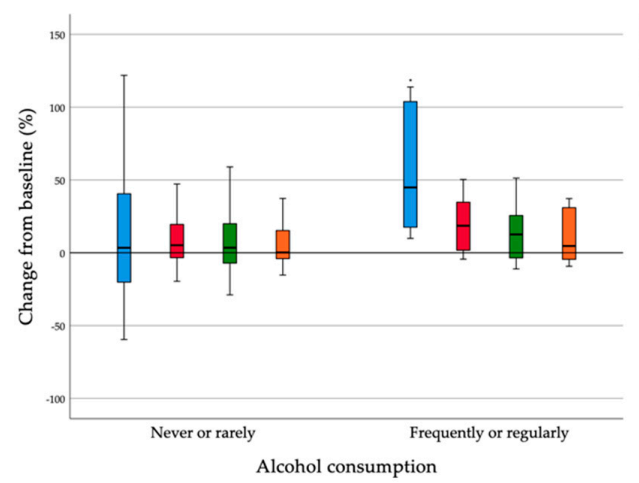

(b)

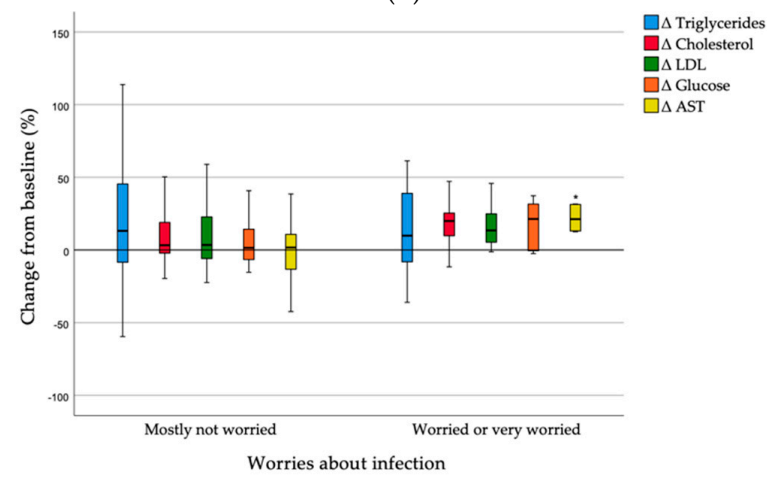

(d)

Figure 2. (a) $\Delta$ serum markers in groups with different outdoor activities during lockdown; (b) $\Delta$ serum markers in groups with different alcohol consumption during lockdown; (c) $\Delta$ serum markers in groups with different social interactions during lockdown; (d) $\Delta$ serum markers in groups with different levels of worries about infection during lockdown. $\Delta$-change of post-lockdown value from baseline; LDL—low-density lipoprotein; AST—aspartate transaminase; HH-household. ${ }^{*} p<0.05$ is considered statistically significant. 
Higher vegetable per energy unit intake $(p=0.028)$ and lower dairy $(p=0.042)$ and total protein intake per energy unit $(p=0.023)$ were detected in those who had social interaction only with family in the same household compared to those who had social interactions also outside their household. Furthermore, higher greens and beans per energy unit intake $(p=0.037)$ and lower refined grains intake were found in those who were very worried about infection than in those who were not.

\subsection{Correlations between Food Quality and Serum Marker Changes Due to Lockdown}

Furthermore, we investigated how the observed changes in serum parameters were associated to the changes in dietary habits. We observed a statistically significant negative association between $\Delta$ HEI and $\Delta$ serum glucose $(\mathrm{r}=-0.328, p=0.044)$. Higher increase in energy intake was positively correlated with higher increase in serum glucose $(r=0.336$, $p=0.039$, Figure 3a). Higher increase in part of energy intake in form of saturated fatty acids was negatively correlated with $\Delta$ serum HDL $(\mathrm{r}=-0.322, p=0.049)$, higher increase in dairy intake per $1000 \mathrm{kcal}$ was weakly positively correlated with $\Delta$ serum HDL $(\mathrm{r}=0.0372$, $p=0.022)$ and negatively correlated with $\Delta$ serum LDL $(\mathrm{r}=-0.385, p=0.017$, Figure $3 \mathrm{~b})$ and higher increase in total protein food intake per $1000 \mathrm{kcal}$ was positively correlated with $\Delta$ serum HDL $(r=0.373, p=0.021)$. Higher increase in omega- 6 fatty acids intake was positively correlated with higher increase in serum glucose $(\mathrm{r}=0.390, p=0.016$, Figure 3c). Higher increase in monounsaturated fatty acids intake was inversely correlated with $\Delta$ serum LDL $(r=-0.356, p=0.028$, Figure $3 \mathrm{~d})$.

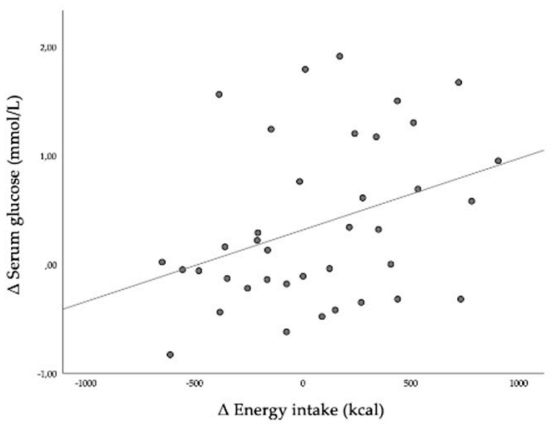

(a)

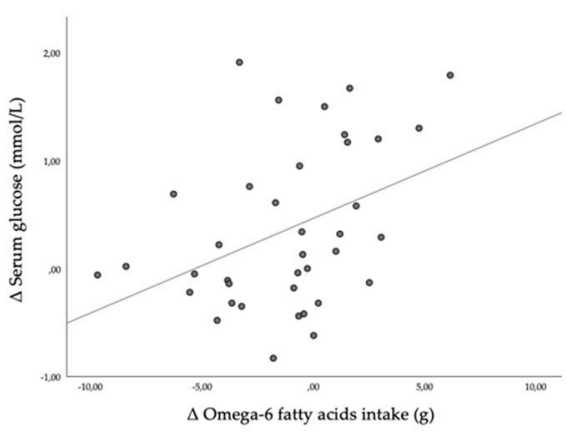

(c)

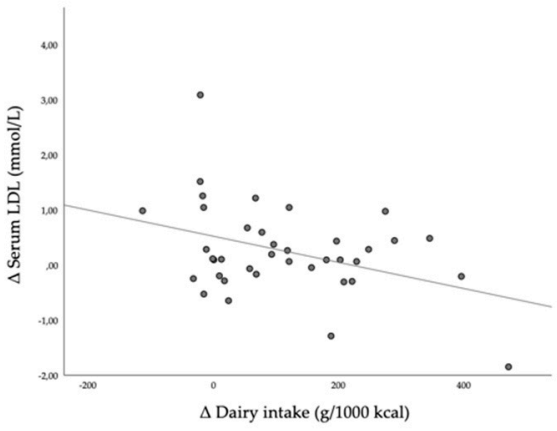

(b)

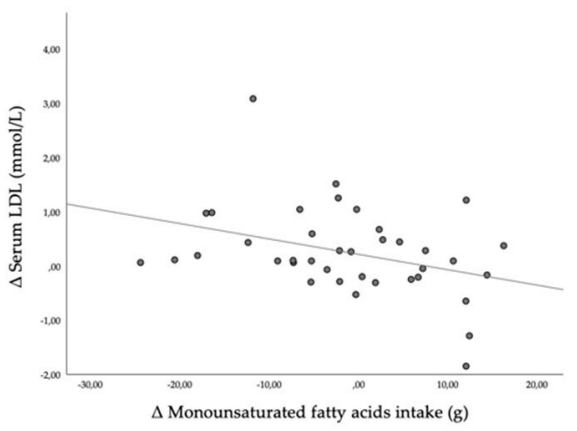

(d)

Figure 3. (a) Correlation between $\Delta$ energy intake and $\Delta$ serum glucose; (b) correlation between $\Delta$ dairy intake and $\Delta$ serum LDL; (c) correlation between $\Delta$ omega-6 fatty acids intake and $\Delta$ serum glucose; (d) correlation between $\Delta$ monounsaturated fatty acids intake and $\Delta$ serum LDL; $\Delta$ 一 change of post-lockdown value from baseline.

\section{Discussion}

This is the first study investigating the impact of COVID-19 lockdown on changes in lifestyle, nutrition and serum markers in healthy adults. Despite a small number of participants, we observed some interesting and potentially important results. There was a 
transient decrease in diet quality during lockdown, whereas post-lockdown general diet quality returned to near baseline. The subjects reported significantly decreased physical activity during lockdown and a significantly decreased energy intake that returned to baseline post-lockdown. Subjects did not report changes in body mass during lockdown, nor did we observe any change in body composition from baseline in the post-lockdown measurement. Serum markers, however, changed significantly post-lockdown, which may point to a possible impact of lockdown on healthy adults' health markers.

Blood sample analysis showed a significant increase in glucose, total cholesterol and LDL levels and a decrease in CRP levels post-lockdown in our participants. The mean value of serum CRP levels was below $1 \mathrm{mg} / \mathrm{L}$ (the Slovenian reference values are $0-8 \mathrm{mg} / \mathrm{L}$ ), which is extremely low and the change from baseline to post-lockdown was $-0.306 \mathrm{mg} / \mathrm{L}$, which was a minor change, even though it was statistically significant. Total cholesterol and LDL mean values post-lockdown exceeded Slovenian reference values. Higher serum glucose, total cholesterol and LDL levels are risk factors for developing metabolic syndrome [22] and each $1 \mathrm{mmol} / \mathrm{L}$ increase in serum cholesterol significantly increases the risk for all-cause mortality in all age groups [23]. A meta-analysis showed the total cholesterol/HDL ratio is the strongest predictor of mortality from ischemic heart disease [24]; the ratio in our study significantly increased from 2.8 to $3.9(p<0.001)$, which could implicate a higher risk for heart disease among subjects in the long term. An increase in glucose of $0.361 \mathrm{mmol} / \mathrm{L}$ was observed. Increase in serum glucose of $1 \mathrm{mmol} / \mathrm{L}$ significantly increased the risk for all-cause mortality in a prospective study and this effect was stronger in younger adults [25].

Higher increases in serum glucose levels were observed in healthy subjects who were worried or very worried about infection with SARS-CoV-2 compared to subjects who were mostly not worried about infection, even though the changes were not statistically significant. Increases in serum glucose levels post-lockdown among subjects that were socially interacting with work colleagues and friends, however, were significantly higher than increases in serum glucose levels in those who were socially interacting only with family in the same household. The subjects who had more social interactions due to work were potentially exposed to a number of risky contacts and therefore were possibly more concerned, experienced more stress and had higher glucose levels. Being worried or experiencing more stress leads to the release of stress hormones such as cortisol, which increases serum glucose levels and influences serum cholesterol levels [26]. We also observed higher increase in serum AST levels among subjects that were worried or very worried about infection with SARS-CoV-2 and some trends for higher increase in serum cholesterol and LDL, but the changes were not statistically significant. Significantly higher increases in serum glucose levels post-lockdown were observed among subjects that were active only in the close proximity to home than among subjects that were active in nature or woods during lockdown. A previous study found that lockdown significantly affected mental health, while contact with nature (blue-green spaces) helped people to cope with these impacts [27], which may explain lower increase in serum glucose levels among subjects that were spending more time in nature.

The literature reports the impact of lockdown on glycaemic control only among patients with diabetes mellitus type 1 and 2 . A retrospective study compared glycaemic control of patients with type 1 diabetes mellitus during lockdown to the same time period in the previous year. They found it was significantly worse during lockdown and the use of insulin therapy was significantly higher, which the authors ascribe to the reduced physical activity, changed dietary habits and higher levels of anxiety during lockdown [28]. Similar was found in a study by Barchetta et al. [29]. A study among patients with well-controlled type 2 diabetes mellitus showed worse glucose control during lockdown, which was worse among patients with higher fasting triglyceride levels pre-lockdown [30]. On the contrary, some studies found better glycaemic control in patients with type 1 diabetes mellitus during lockdown, which could be due to having more time for self-management during lockdown, at least in the short term [29,31-33] or due to reduced work-related stress [34]. 
In the present study, we found a decrease in HEI during lockdown. Decreased intake of total protein food, seafood and plant proteins per energy unit, increased intake of refined grains and worse composition of fatty acids in the diet (decreased PUFA+MUFA)/SFA) contributed to this drop. HEI has been associated with lower risk factors for development of chronic noncommunicable diseases, such as cardiovascular diseases, cancer and all-cause mortality [35]. It has also been associated with improved low-grade inflammation [36]. The literature reports different changes in diet quality during lockdown. A small increase in HEI during early lockdown compared with baseline was found in a Canadian study in which the authors hypothesized that eating out less often may explain such an observation [37]. A Spanish survey also observed increased adherence to the Mediterranean diet, which points to a better diet quality during lockdown [38]. On the other hand, one of the Italian surveys did not detect any changes in dietary habits and more than a third of the subjects stated their diet worsened during lockdown [39], while another found a decrease in diet quality [40].

Post-lockdown HEI of our participants returned to nearly baseline levels, but vegetable, greens and beans, total protein and seafood and plant protein intake per energy unit were still significantly lower post-lockdown, while refined grains intake per energy unit and \% energy intake in form of saturated fats intake were still significantly higher. Additionally, sodium intake per energy unit increased post-lockdown. Interestingly, the participants who were worried about infection increased vegetable intake per energy unit post lockdown and lowered refined grains per energy unit intake more than those who were not worried about infection. Higher $\Delta$ vegetable intake was observed also in those who had social interaction only with family in the same household compared to those who had social interactions also outside their household. An increase in starch and dietary fibre intake was observed in all participants. Post-lockdown dairy intake per energy unit increased and added sugars intake per energy unit decreased, which contributed to a higher HEI at post-lockdown than during lockdown. We observed a statistically significant negative association between $\Delta$ HEI and $\Delta$ serum glucose. Increase in dairy intake per energy unit positively correlated to $\Delta$ serum HDL and negatively correlated to $\Delta$ serum LDL, which is in line with reported positive association between dairy intake and lipid profile [41]. We also observed a correlation between increase in total protein food per energy unit intake and $\Delta$ serum HDL. The ratio of fatty acids intake worsened and saturated fatty acids intake and saturated fatty acids per energy unit were significantly higher post-lockdown. It is known that an increase in saturated fatty acids intake is associated with increased serum total cholesterol and LDL [42]. In our study, we observed an increase in serum total cholesterol and LDL, but the associations between $\Delta$ serum total cholesterol and $\Delta$ serum LDL and $\Delta$ saturated fatty acid intake were not statistically significant; however, higher increase in saturated fatty acids per energy unit was significantly negatively correlated with $\Delta$ serum HDL. It has been shown that an increase in saturated fatty acids intake could lead to a temporary insulin resistance and consequently higher serum glucose levels [43]. An increase in serum glucose was indeed observed, but there were no associations between $\Delta$ serum glucose and $\Delta$ saturated fatty acid intake. We observed a negative association between $\Delta$ monounsaturated fatty acids intake and $\Delta$ LDL. The intake of omega- 6 fatty acids decreased and consequently the omega-3/omega- 6 fatty acids ratio increased. Despite this, we observed a statistically significant association between $\Delta$ omega- 6 fatty acids intake and $\Delta$ serum glucose. It should be noted, however, that the aforementioned correlations were weak to moderate.

Energy intake decreased during lockdown, but no changes in energy intake between baseline and post-lockdown were observed, even though there was a decrease in energy density. Our participants also maintained a constant number of meals during lockdown. Nevertheless, there was a statistically significant association between increased energy intake and increased serum glucose. Contrary to our study, other studies reported an increased energy intake during lockdown [44], an increased number of meals per day [45] and increased consumption of homemade pastries and fried food [46], which may lead 
to increased energy intake [47]. An increased snacking [46], especially in sweet snacks intake was also observed [44,45,48]. Moreover, studies also reported an increase in snacking between meals and late-night snacking, especially an increase in the intake of salty snacks [45,49]. In one study, almost half of the subjects felt anxious about their eating habits and stated they consumed more food, particularly comfort foods with the aim to feel better [50]. We observed a trend in increased alcohol intake during lockdown that was not statistically significant (data not shown) but did not observe any changes in alcohol intake between post-lockdown and baseline. This is in line with a previous report, where $70 \%$ of the subjects reported no changes in their alcohol intake during lockdown [46]. However, significantly higher increase in serum triglyceride levels was observed among subjects that frequently or regularly consumed alcohol during lockdown than those that never or rarely consumed alcohol, pointing to a known fact that excessive alcohol intake may cause hypertriglyceridemia postprandially and in the fasting state [51]. An increase in alcohol intake was observed in some other studies [44,48].

The subjects' physical activity levels were lower during lockdown, which was reported also in other studies [39-41,52]. One study [44] reported increased screen time in half of the subjects, which could be one reason for lower physical activity levels; other reasons include closed sport facilities, restricted outdoor activities and less physical activity due to work and transport. An online survey also found more than a third of the subjects did not engage in any form of physical activity and they also reported five or more hours of screen time per day [53]. On the contrary, an Italian survey reported no changes in physical activity during lockdown [40]. Decreased physical activity itself is a risk factor for low-grade inflammation and development of metabolic disorders [54]; however, despite lower physical activity levels, we did not observe any increase in inflammatory marker CRP. The observed increase in fasting serum glucose could be partially due to decreased physical activity, as the association was shown before [55].

Despite lower physical activity levels during lockdown, we did not observe any significant changes in body mass or body composition, probably due to a lower energy intake during lockdown. Maintenance in body mass in our study is contrary to most other studies, which reported an increase in body mass during lockdown $[37,46,53,56,57]$, although there are also studies with observations similar to ours in patients with type 2 diabetes mellitus [58], and in healthy subjects [40]. Interestingly, it was observed that overweight and obese subjects gained body mass, while underweight subjects lost it [48]. Our subjects all had normal body mass at baseline.

There are limitations to our study. The sample size (38 subjects) is small. The subjects dropped-out because of their fear of infection with SARS-CoV-2 while taking part in the measurements at our faculty. While the sample was big enough for evaluating changes in serum parameters (statistical power of primary outcome, $\Delta$ serum glucose was 0.826 ), a bigger sample would be needed to thoroughly evaluate changes in lifestyle. Due to lockdown restrictions, we could not perform lockdown measurement in our facility, therefore for that timepoint we only have data that could be collected online with questionnaires. The subjects were healthy volunteers who had an interest in nutrition and were already engaging in healthy behaviours, so the sample may not be representative for the general population. Nevertheless, our results provide some insight into the impact of lockdown on health markers in asymptomatic subjects with normal body mass, which was very scarce before.

Social distancing and psychological stress due to the COVID-19 pandemic have been reported to have led to harmful health behaviours that are associated with noncommunicable diseases and can interfere with immunity [59]. Moreover, managing lifestyle risk factors, such as diet, physical activity, smoking and alcohol consumption can prevent severe COVID-19 outcomes [60]. Impaired glucose control and dyslipidaemia have also been demonstrated to be risk factors for worse COVID-19 outcomes [61-63]. We observed a worsened serum biomarkers profile after lockdown among healthy subjects with a normal body mass, healthy lifestyle and eating patterns. Despite observed changes in diet 
quality, changes in serum biomarkers could not be explained by them. Our participants maintained similar energy intake post-lockdown to background, their omega- 6 fatty acids intake decreased, while total protein food intake per energy unit and dairy intake increased. Observed moderate correlations between aforementioned changes in nutrition and changes in serum markers point to a possible small positive effect of nutritional changes on serum markers. There was, however, an increase in serum glucose, total cholesterol and LDL post-lockdown. Furthermore, we observed increased dietary fibre and total ORAC intake and decreased added sugar intake, which have all been reported to have favourable effects on serum glucose and cholesterol [64]. Increase in serum glucose with the duration of confinement was reported in a study of 520-day mission simulation to Mars [65]. Effects of factors such as worry, stress and confinement on serum markers could be taken into account for the observed changes in serum markers. The fact that the improvement of certain healthy behaviours observed in our small study did not counterbalance the effect of stress is an important public health aspect that should be further explored.

\section{Conclusions}

This is the first study investigating the changes in diet and physical activity during lockdown and their effect on serum markers in healthy adults. Significant changes in serum markers were observed after only 3 months of lockdown, such as an increase in serum glucose, total cholesterol, and LDL levels. Such changes increase the risk for developing metabolic syndrome, heart disease and all-cause mortality. The changes of serum markers therefore point to a possible impact of lockdown on health markers.

Author Contributions: Conceptualization, N.M. and Z.J.P.; methodology, N.B.J., K.N., S.K., A.P., Z.J.P., N.M.; software, K.N., N.B.J.; validation, N.B.J.; formal analysis, K.N., N.B.J.; investigation, N.B.J., K.N., S.K., A.P., Z.J.P., N.M.; resources, Z.J.P.; data curation, N.B.J., K.N.; writing-original draft preparation, N.B.J., K.N.; writing-review and editing, N.M., Z.J.P., S.K., A.P.; visualization, N.B.J., K.N.; supervision, N.M., Z.J.P.; project administration, N.M., Z.J.P. All authors have read and agreed to the published version of the manuscript.

Funding: The study was supported by the Slovenian Research Agency (Programme P1-0386 and I0-0035).

Institutional Review Board Statement: The study was conducted according to the guidelines of the Declaration of Helsinki and approved by the Slovenian National Medical Ethics Committee (No. 0120-557/2017/4) and was registered on ClinicalTrials.gov (Identifier: NCT04347213).

Informed Consent Statement: Informed consent was obtained from all subjects involved in the study.

Data Availability Statement: The data presented in this study are available on request from the corresponding author.

Acknowledgments: Authors thank the participants for participating in the study and their readiness to collaborate with us during lockdown and after it.

Conflicts of Interest: The authors declare no conflict of interest.

\section{References}

1. WHO. WHO Director-General's Opening Remarks at the Media Briefing on COVID-19-11 March 2020. Available online: https:/ / www.who.int/dg/speeches/detail/who-director-general-s-opening-remarks-at-the-media-briefing-on-covid-19---1 1-march-2020 (accessed on 9 April 2020).

2. Sandford, A. Coronavirus: Half of Humanity on Lockdown in 90 Countries. Available online: https://www.euronews.com/20 20/04/02/ coronavirus-in-europe-spain-s-death-toll-hits-10-000-after-record-950-new-deaths-in-24-hou (accessed on 12 May 2020).

3. Rehman, H.; Ahmad, M.I. COVID-19: Quarantine, Isolation, and Lifestyle Diseases. Arch. Physiol. Biochem. 2020. [CrossRef] [PubMed]

4. Vlada Republike Slovenije. Slovenija Razglasila Epidemijo Novega Koronavirusa. Available online: https://www.gov.si/novice/ 2020-03-12-slovenija-razglasila-epidemijo-novega-koronavirusa/ (accessed on 18 June 2020). 
5. Odlok o Preklicu Epidemije Nalezljive Bolezni SARS-CoV-2 (COVID-19)/Ordinance on the Revocation of the COVID-19 Epidemic. Uradni list RS, št. 68/20. Available online: http:/ / pisrs.si (accessed on 25 January 2021).

6. Cetron, M.; Maloney, S.; Koppaka, R.; Simone, P. Isolation and Quarantine: Containment Strategies for SARS 2003; National Academies Press: Washington, DC, USA, 2004.

7. Dignum, F.; Dignum, V.; Davidsson, P.; Ghorbani, A.; van der Hurk, M.; Jensen, M.; Kammler, C.; Lorig, F.; Ludescher, L.G.; Melchior, A.; et al. Analysing the Combined Health, Social and Economic Impacts of the Corovanvirus Pandemic Using Agent-Based Social Simulation. Minds Mach. 2020, 1-18. [CrossRef] [PubMed]

8. Pearl, R.L. Weight Stigma and the "Quarantine-15". Obesity 2020. [CrossRef]

9. Abbas, A.M.; Kamel, M.M. Dietary Habits in Adults during Quarantine in the Context of COVID-19 Pandemic. Obes Med. 2020, 19, 100254. [CrossRef]

10. Mattioli, A.V.; Ballerini Puviani, M.; Nasi, M.; Farinetti, A. COVID-19 Pandemic: The Effects of Quarantine on Cardiovascular Risk. Eur. J. Clin. Nutr. 2020, 74, 852-855. [CrossRef]

11. Dramatično Stanje v Izropanih Slovenskih Trgovinah. Available online: https://novice.svet24.si/clanek/novice/slovenija/5e6a8 3b45c265/ foto-dramaticno-stanje-v-izropanih-slovenskih-trgovinah-police-prazne-roba-v-skladiscih (accessed on 9 April 2020).

12. Childs, C.E.; Calder, P.C.; Miles, E.A. Diet and Immune Function. Nutrients 2019, 11, 1933. [CrossRef]

13. Noncommunicable Diseases: Risk Factors. Available online: https://www.who.int/data/gho/data/themes/topics/topicdetails/GHO/ncd-risk-factors (accessed on 1 March 2021).

14. Eckel, R.H.; Alberti, K.; Grundy, S.M.; Zimmet, P.Z. The Metabolic Syndrome. Lancet 2010, 375, 181-183. [CrossRef]

15. Bizjak, M.; Jenko-Pražnikar, Z.; Koroušić Seljak, B. Development and Validation of an Electronic FFQ to Assess Food Intake in the Slovene Population. Public Health Nutr. 2014, 17, 1729-1737. [CrossRef] [PubMed]

16. Korošec, M.; Golob, T.; Bertoncelj, J.; Stibilj, V.; Seljak, B. The Slovenian Food Composition Database. Food Chem. 2013, 140, 495-499. [CrossRef]

17. Souci, S.W.; Fachmann, W.; Kraut, H. Food Composition and Nutrition Tables; Medpharm: Centurion, South Africa, 2000.

18. Reedy, J.; Lerman, J.L.; Krebs-Smith, S.M.; Kirkpatrick, S.I.; Pannucci, T.E.; Wilson, M.M.; Subar, A.F.; Kahle, L.L.; Tooze, J.A. Evaluation of the Healthy Eating Index-2015. J. Acad. Nutr. Diet. 2018, 118, 1622-1633. [CrossRef]

19. Center for Nutrition Policy and Promotion. How the HEI Is Scored I USDA-FNS. Available online: https://www.fns.usda.gov/ how-hei-scored (accessed on 30 July 2020).

20. Craig, C.L.; Marshall, A.L.; Sjöström, M.; Bauman, A.E.; Booth, M.L.; Ainsworth, B.E.; Pratt, M.; Ekelund, U.; Yngve, A.; Sallis, J.F.; et al. International Physical Activity Questionnaire: 12-Country Reliability and Validity. Med. Sci. Sports Exerc. 2003, 35, 1381-1395. [CrossRef]

21. Tuck, M.K.; Chan, D.W.; Chia, D.; Godwin, A.K.; Grizzle, W.E.; Krueger, K.E.; Rom, W.; Sanda, M.; Sorbara, L.; Stass, S.; et al. Standard Operating Procedures for Serum and Plasma Collection: Early Detection Research Network Consensus Statement Standard Operating Procedure Integration Working Group. J. Proteome Res. 2009, 8, 113-117. [CrossRef]

22. Huang, P.L. A Comprehensive Definition for Metabolic Syndrome. Dis. Models Mech. 2009, 2, 231-237. [CrossRef] [PubMed]

23. Yi, S.-W.; Yi, J.-J.; Ohrr, H. Total Cholesterol and All-Cause Mortality by Sex and Age: A Prospective Cohort Study among 12.8 Million Adults. Sci. Rep. 2019, 9, 1596. [CrossRef]

24. Lewington, S.; Whitlock, G.; Clarke, R.; Sherliker, P.; Emberson, J.; Halsey, J.; Qizilbash, N.; Peto, R.; Collins, R.; Prospective Studies Collaboration. Blood Cholesterol and Vascular Mortality by Age, Sex, and Blood Pressure: A Meta-Analysis of Individual Data from 61 Prospective Studies with 55,000 Vascular Deaths. Lancet 2007, 370, 1829-1839. [CrossRef] [PubMed]

25. Yi, S.-W.; Park, S.; Lee, Y.; Park, H.-J.; Balkau, B.; Yi, J.-J. Association between Fasting Glucose and All-Cause Mortality According to Sex and Age: A Prospective Cohort Study. Sci. Rep. 2017, 7, 8194. [CrossRef]

26. De Guia, R.M.; Rose, A.J.; Herzig, S. Glucocorticoid Hormones and Energy Homeostasis. Horm. Mol. Biol. Clin. Investig. 2014, 19, 117-128. [CrossRef] [PubMed]

27. Pouso, S.; Borja, Á.; Fleming, L.E.; Gómez-Baggethun, E.; White, M.P.; Uyarra, M.C. Contact with Blue-Green Spaces during the COVID-19 Pandemic Lockdown Beneficial for Mental Health. Sci. Total Environ. 2021, 756, 143984. [CrossRef]

28. Ghesquière, L.; Garabedian, C.; Drumez, E.; Lemaître, M.; Cazaubiel, M.; Bengler, C.; Vambergue, A. Effects of COVID-19 Pandemic Lockdown on Gestational Diabetes Mellitus: A Retrospective Study. Diabetes Metab. 2020, 101201. [CrossRef]

29. Barchetta, I.; Cimini, F.A.; Bertoccini, L.; Ceccarelli, V.; Spaccarotella, M.; Baroni, M.G.; Cavallo, M.G. Effects of Work Status Changes and Perceived Stress Onglycaemiccontrol in Individuals with Type 1 Diabetes during COVID-19 Lockdown in Italy. Diabetes Res. Clin. Pract. 2020, 170, 108513. [CrossRef] [PubMed]

30. Biancalana, E.; Parolini, F.; Mengozzi, A.; Solini, A. Short-Term Impact of COVID-19 Lockdown on Metabolic Control of Patients with Well-Controlled Type 2 Diabetes: A Single-Centre Observational Study. Acta Diabetol. 2020. [CrossRef] [PubMed]

31. Bonora, B.M.; Boscari, F.; Avogaro, A.; Bruttomesso, D.; Fadini, G.P. Glycaemic Control Among People with Type 1 Diabetes During Lockdown for the SARS-CoV-2 Outbreak in Italy. Diabetes 2020, 1-11. [CrossRef]

32. Grabia, M.; Markiewicz-Żukowska, R.; Puścion-Jakubik, A.; Bielecka, J.; Nowakowski, P.; Gromkowska-Kępka, K.; Mielcarek, K.; Socha, K. The Nutritional and Health Effects of the COVID-19 Pandemic on Patients with Diabetes Mellitus. Nutrients 2020, 12, 3013. [CrossRef] [PubMed]

33. Prabhu Navis, J.; Leelarathna, L.; Mubita, W.; Urwin, A.; Rutter, M.K.; Schofield, J.; Thabit, H. Impact of COVID-19 Lockdown on Flash and Real-Time Glucose Sensor Users with Type 1 Diabetes in England. Acta Diabetol. 2020. [CrossRef] 
34. Aragona, M.; Rodia, C.; Bertolotto, A.; Campi, F.; Coppelli, A.; Giannarelli, R.; Bianchi, C.; Dardano, A.; Del Prato, S. Type 1 Diabetes and COVID-19: The "Lockdown Effect". Diabetes Res. Clin. Pract. 2020, 170, 108468. [CrossRef]

35. Onvani, S.; Haghighatdoost, F.; Surkan, P.J.; Larijani, B.; Azadbakht, L. Adherence to the Healthy Eating Index and Alternative Healthy Eating Index Dietary Patterns and Mortality from All Causes, Cardiovascular Disease and Cancer: A Meta-Analysis of Observational Studies. J. Hum. Nutr. Diet. 2017, 30, 216-226. [CrossRef] [PubMed]

36. Barbaresko, J.; Koch, M.; Schulze, M.B.; Nöthlings, U. Dietary Pattern Analysis and Biomarkers of Low-Grade Inflammation: A Systematic Literature Review. Nutr. Rev. 2013, 71, 511-527. [CrossRef]

37. Lamarche, B.; Brassard, D.; Lapointe, A.; Laramée, C.; Kearney, M.; Côté, M.; Bélanger-Gravel, A.; Desroches, S.; Lemieux, S.; Plante, C. Changes in Diet Quality and Food Security among Adults during the COVID-19-Related Early Lockdown: Results from NutriQuébec. Am. J. Clin. Nutr. 2021. [CrossRef]

38. Rodríguez-Pérez, C.; Molina-Montes, E.; Verardo, V.; Artacho, R.; García-Villanova, B.; Guerra-Hernández, E.J.; Ruíz-López, M.D. Changes in Dietary Behaviours during the COVID-19 Outbreak Confinement in the Spanish COVIDiet Study. Nutrients 2020, 12, 1730. [CrossRef]

39. Di Renzo, L.; Gualtieri, P.; Pivari, F.; Soldati, L.; Attinà, A.; Cinelli, G.; Leggeri, C.; Caparello, G.; Barrea, L.; Scerbo, F.; et al. Eating Habits and Lifestyle Changes during COVID-19 Lockdown: An Italian Survey. J. Transl. Med. 2020, 18, 229. [CrossRef]

40. Cicero, A.F.G.; Fogacci, F.; Giovannini, M.; Mezzadri, M.; Grandi, E.; Borghi, C.; The Brisighella Heart Study Group. COVID19-Related Quarantine Effect on Dietary Habits in a Northern Italian Rural Population: Data from the Brisighella Heart Study. Nutrients 2021, 13, 309. [CrossRef]

41. Anto, L.; Warykas, S.W.; Torres-Gonzalez, M.; Blesso, C.N. Milk Polar Lipids: Underappreciated Lipids with Emerging Health Benefits. Nutrients 2020, 12, 1001. [CrossRef]

42. Hooper, L.; Martin, N.; Abdelhamid, A.; Smith, G.D. Reduction in Saturated Fat Intake for Cardiovascular Disease. Cochrane Database Syst. Rev. 2015. [CrossRef] [PubMed]

43. Koska, J.; Ozias, M.K.; Deer, J.; Kurtz, J.; Salbe, A.D.; Harman, S.M.; Reaven, P.D. A Human Model of Dietary Saturated Fatty Acid Induced Insulin Resistance. Metab. Clin. Exp. 2016, 65, 1621-1628. [CrossRef] [PubMed]

44. Górnicka, M.; Drywień, M.E.; Zielinska, M.A.; Hamułka, J. Dietary and Lifestyle Changes During COVID-19 and the Subsequent Lockdowns among Polish Adults: A Cross-Sectional Online Survey PLifeCOVID-19 Study. Nutrients 2020, 12, 2324. [CrossRef]

45. Błaszczyk-Bębenek, E.; Jagielski, P.; Bolesławska, I.; Jagielska, A.; Nitsch-Osuch, A.; Kawalec, P. Nutrition Behaviors in Polish Adults before and during COVID-19 Lockdown. Nutrients 2020, 12, 3084. [CrossRef]

46. Kriaucioniene, V.; Bagdonaviciene, L.; Rodríguez-Pérez, C.; Petkeviciene, J. Associations between Changes in Health Behaviours and Body Weight during the COVID-19 Quarantine in Lithuania: The Lithuanian COVIDiet Study. Nutrients 2020, $12,3119$. [CrossRef] [PubMed]

47. Kahleova, H.; Lloren, J.I.; Mashchak, A.; Hill, M.; Fraser, G.E. Meal Frequency and Timing Are Associated with Changes in Body Mass Index in Adventist Health Study 2. J. Nutr. 2017, 147, 1722-1728. [CrossRef]

48. Sidor, A.; Rzymski, P. Dietary Choices and Habits during COVID-19 Lockdown: Experience from Poland. Nutrients 2020, $12,1657$. [CrossRef]

49. Husain, W.; Ashkanani, F. Does COVID-19 Change Dietary Habits and Lifestyle Behaviours in Kuwait: A Community-Based Cross-Sectional Study. Env. Health Prev. Med. 2020, 25, 61. [CrossRef]

50. Di Renzo, L.; Gualtieri, P.; Cinelli, G.; Bigioni, G.; Soldati, L.; Attinà, A.; Bianco, F.F.; Caparello, G.; Camodeca, V.; Carrano, E.; et al. Psychological Aspects and Eating Habits during COVID-19 Home Confinement: Results of EHLC-COVID-19 Italian Online Survey. Nutrients 2020, 12, 2152. [CrossRef]

51. Van de Wiel, A. The Effect of Alcohol on Postprandial and Fasting Triglycerides. Int. J. Vasc. Med. 2012. [CrossRef]

52. Ruíz-Roso, M.B.; de Carvalho Padilha, P.; Matilla-Escalante, D.C.; Brun, P.; Ulloa, N.; Acevedo-Correa, D.; Arantes Ferreira Peres, W.; Martorell, M.; Rangel Bousquet Carrilho, T.; de Oliveira Cardoso, L.; et al. Changes of Physical Activity and Ultra-Processed Food Consumption in Adolescents from Different Countries during Covid-19 Pandemic: An Observational Study. Nutrients 2020, 12, 2289. [CrossRef] [PubMed]

53. Cheikh Ismail, L.; Osaili, T.M.; Mohamad, M.N.; Al Marzouqi, A.; Jarrar, A.H.; Abu Jamous, D.O.; Magriplis, E.; Ali, H.I.; Al Sabbah, H.; Hasan, H.; et al. Eating Habits and Lifestyle during COVID-19 Lockdown in the United Arab Emirates: A Cross-Sectional Study. Nutrients 2020, 12, 3314. [CrossRef]

54. Biswas, A.; Oh, P.I.; Faulkner, G.E.; Bajaj, R.R.; Silver, M.A.; Mitchell, M.S.; Alter, D.A. Sedentary Time and Its Association with Risk for Disease Incidence, Mortality, and Hospitalization in Adults: A Systematic Review and Meta-Analysis. Ann. Intern. Med. 2015, 162, 123-132. [CrossRef]

55. Kujala, U.M.; Mäkinen, V.-P.; Heinonen, I.; Soininen, P.; Kangas, A.J.; Leskinen, T.H.; Rahkila, P.; Würtz, P.; Kovanen, V.; Cheng, S.; et al. Long-Term Leisure-Time Physical Activity and Serum Metabolome. Circulation 2013, 127, 340-348. [CrossRef] [PubMed]

56. Pellegrini, M.; Ponzo, V.; Rosato, R.; Scumaci, E.; Goitre, I.; Benso, A.; Belcastro, S.; Crespi, C.; De Michieli, F.; Ghigo, E.; et al. Changes in Weight and Nutritional Habits in Adults with Obesity during the "Lockdown" Period Caused by the COVID-19 Virus Emergency. Nutrients 2020, 12, 2016. [CrossRef] [PubMed]

57. Barrea, L.; Pugliese, G.; Framondi, L.; Di Matteo, R.; Laudisio, D.; Savastano, S.; Colao, A.; Muscogiuri, G. Does Sars-Cov-2 Threaten Our Dreams? Effect of Quarantine on Sleep Quality and Body Mass Index. J. Transl. Med. 2020, 18, 318. [CrossRef] [PubMed] 
58. Önmez, A.; Gamsızkan, Z.; Özdemir, Ș.; Kesikbaş, E.; Gökosmanoğlu, F.; Torun, S.; Cinemre, H. The Effect of COVID-19 Lockdown on Glycemic Control in Patients with Type 2 Diabetes Mellitus in Turkey. Diabetes Metab. Syndr. Clin. Res. Rev. 2020, 14, 1963-1966. [CrossRef]

59. Lange, K.W.; Nakamura, Y. Lifestyle Factors in the Prevention of COVID-19. Glob. Health J. 2020, 4, 146-152. [CrossRef]

60. Hamer, M.; Kivimäki, M.; Gale, C.R.; Batty, G.D. Lifestyle Risk Factors, Inflammatory Mechanisms, and COVID-19 Hospitalization: A Community-Based Cohort Study of 387,109 Adults in UK. Brain Behav. Immun. 2020, 87, 184-187. [CrossRef] [PubMed]

61. Hariyanto, T.I.; Kurniawan, A. Dyslipidemia Is Associated with Severe Coronavirus Disease 2019 (COVID-19) Infection. Diabetes Metab. Syndr. 2020, 14, 1463-1465. [CrossRef]

62. Erener, S. Diabetes, Infection Risk and COVID-19. Mol. Metab. 2020, 39, 101044. [CrossRef]

63. Apicella, M.; Campopiano, M.C.; Mantuano, M.; Mazoni, L.; Coppelli, A.; Prato, S.D. COVID-19 in People with Diabetes: Understanding the Reasons for Worse Outcomes. Lancet Diabetes Endocrinol. 2020, 8, 782-792. [CrossRef]

64. De la Iglesia, R.; Loria-Kohen, V.; Zulet, M.A.; Martinez, J.A.; Reglero, G.; Ramirez de Molina, A. Dietary Strategies Implicated in the Prevention and Treatment of Metabolic Syndrome. Int. J. Mol. Sci. 2016, 17, 1877. [CrossRef] [PubMed]

65. Strollo, F.; Macchi, C.; Eberini, I.; Masini, M.A.; Botta, M.; Vassilieva, G.; Nichiporuk, I.; Monici, M.; Santucci, D.; Celotti, F.; et al. Body Composition and Metabolic Changes during a 520-Day Mission Simulation to Mars. J. Endocrinol. Investig. 2018, 41, 1267-1273. [CrossRef] [PubMed] 